\title{
Quantization of bosonic fields with two mass and spin states
}

\author{
S. I. Kruglov \\ University of Toronto at Scarborough, \\ Physical and Environmental Sciences Department, \\ 1265 Military Trail, Toronto, Ontario, Canada M1C 1 A4
}

\begin{abstract}
We investigate bosonic fields possessing two mass and spin states. The density matrix in the first order formalism is obtained. The quantization of fields in the first order formulation is performed and propagators are found.
\end{abstract}

Pacs: 03.65.Pm, 03.70.+k, 04.20.Fy, 11.10.-z

\section{Introduction}

The theory of massive vector fields play very important role in the standard electroweak model. We mention also that propagating torsion axial vector fields appear in quantum gravity [1]. These fields possess two mass and spin states [2]. The classical theory of bosonic fields with two mass and spin states was considered in 3 . Here we quantize such fields in the first order formalism.

The paper is organized as follows. In Sec.2 the propagator of the bosonic fields with two mass and spin states in the second order formulation is obtained. The first order wave equation for fields in the $11 \times 11$-matrix form is investigated in Sec.3. We obtain the minimal equation for the $11 \times 11$ matrices and the density matrix. The quantization of the field is discussed in Sec.4. The commutation relations and the vacuum expectation of chronological pairing of operators are found. A conclusion is given in Sec.5.

We use the Euclidian metric and the four-vector of the field is $B_{\mu}=$ $\left(B_{m}, B_{4}\right) ; m=1,2,3 ; B_{4}=i B_{0}$. 


\section{$2 \quad$ Field equations}

It the work [3], we considered charged bosonic fields which possess two spins, one and zero, with different masses. The corresponding field equations are

$$
\partial_{\mu}^{2} B_{\nu}+\left(\frac{m^{2}}{m_{0}^{2}}-1\right) \partial_{\nu}\left(\partial_{\alpha} B_{\alpha}\right)-m^{2} B_{\nu}=0
$$

where $m, m_{0}$ are masses of spin- 1 and spin-0 states, correspondingly. For the real functions $\left(\mathbf{B}, B_{0}\right)$ Eq.(1) describes torsion fields that appear in higher derivative quantum gravity [2]. Eq.(1) can be represented, in momentum space, as $4 \times 4$-matrix equation:

$$
M_{\mu \nu} B_{\nu}=0, \quad M_{\mu \nu}=\left(p^{2}+m^{2}\right) \delta_{\mu \nu}+\left(\frac{m^{2}}{m_{0}^{2}}-1\right) p_{\mu} p_{\nu} .
$$

To have non-trivial solutions of Eq.(1), one gets $\operatorname{det} M=0$. This equation leads to the dispersion equation [3]:

$$
\left(p^{2}+m^{2}\right)\left(p^{2}+m_{0}^{2}\right)=0
$$

where the four-momentum being $p_{\mu}=\left(\mathbf{p}, i p_{0}\right)$.

The propagator of fields is defined as $\left(M_{\mu \nu}\right)^{-1}$. We obtain

$$
\left(M_{\mu \nu}\right)^{-1}=\frac{p^{2}+m_{0}^{2}+\left(m_{0}^{2} / m^{2}-1\right) p_{\mu} p_{\nu}}{\left(p^{2}+m^{2}\right)\left(p^{2}+m_{0}^{2}\right)}
$$

so that $M_{\mu \alpha}\left(M_{\alpha \nu}\right)^{-1}=\delta_{\mu \nu}$. The propagator (4) corresponds to bosonic fields that have two states with spin-1, mass $m$, and spin-0, mass $m_{0}$. It is convenient to represent the propagator (4) as a sum of two propagators

$$
\left(M_{\mu \nu}\right)^{-1}=\frac{1+\left(1 / m^{2}\right) p_{\mu} p_{\nu}}{p^{2}+m^{2}}-\frac{\left(1 / m^{2}\right) p_{\mu} p_{\nu}}{p^{2}+m_{0}^{2}} .
$$

The first term in Eq.(5) is the propagator of the Proca equation which can be obtained from Eq.(1) at $m_{0} \rightarrow \infty$. At this limit $\left(m_{0} \rightarrow \infty\right)$ the second term in Eq.(5) vanishes and, therefore, corresponds to the contribution of spin-0 state. The second term in Eq.(5) has a "wrong" sign (-) that indicates on the presence of a ghost. The ghost results in the negative contribution to the energy, and we should introduce indefinite metrics in quantum field theory [4. Therefore, the state with spin-0 and mass $m_{0}$ may be considered as a ghost and can be removed at the limit $m_{0} \rightarrow \infty$. We may treat, therefore, Eq.(1) as the equation for the vector fields in the general gage [5] (see also references therein). 


\section{The first order wave equation}

In [3], Eq.(1) was represented in 11-component matrix form as follows:

$$
\begin{gathered}
\left(\alpha_{\mu} \partial_{\mu}+m P_{1}+\frac{m_{0}^{2}}{m} P_{0}\right) \Psi(x)=0, \\
\Psi(x)=\left\{\psi_{A}(x)\right\}=\left(\begin{array}{c}
(1 / m) B(x) \\
B_{\mu}(x) \\
(1 / m) B_{\mu \nu}(x)
\end{array}\right),
\end{gathered}
$$

where $B_{\mu \nu}=\partial_{\mu} B_{\nu}-\partial_{\nu} B_{\mu}, B(x)=-\left(m^{2} / m_{0}^{2}\right) \partial_{\mu} B_{\mu}(x)$. Matrices entering Eq.(6), expressed trough the elements of the entire matrix algebra $\varepsilon^{A, B}$, are given by

$$
\begin{gathered}
\alpha_{\mu}=\beta_{\mu}^{(1)}+\beta_{\mu}^{(0)}, \quad \beta_{\mu}^{(1)}=\varepsilon^{\nu,[\nu \mu]}+\varepsilon^{[\nu \mu], \nu}, \quad \beta_{\mu}^{(0)}=\varepsilon^{\mu, 0}+\varepsilon^{0, \mu}, \\
P_{1}=\varepsilon^{\mu, \mu}+\frac{1}{2} \varepsilon^{[\mu \nu],[\mu \nu]}, \quad P_{0}=\varepsilon^{0,0} .
\end{gathered}
$$

It is convenient to consider the equivalent form of Eq.(6). Multiplying Eq.(6) by the matrix

$$
M\left(m P_{1}+\frac{m_{0}^{2}}{m} P_{0}\right)^{-1}=M\left(\frac{1}{m} P_{1}+\frac{m}{m_{0}^{2}} P_{0}\right),
$$

where

$$
M=\frac{m m_{0}}{m+m_{0}}
$$

is a reduced mass, and using the properties of the projection operators $P_{1}^{2}=$ $P_{1}, P_{0}^{2}=P_{0}$, we arrive at the standard form of the first order relativistic wave equation

$$
\left(\Gamma_{\mu} \partial_{\mu}+M\right) \Psi(x)=0 .
$$

The matrices of Eq.(10) are given by

$$
\Gamma_{\mu}=\frac{m_{0}}{m+m_{0}}\left(\varepsilon^{\mu, 0}+\varepsilon^{\nu,[\nu \mu]}+\varepsilon^{[\nu \mu], \mu}\right)+\frac{m^{2}}{m_{0}\left(m+m_{0}\right)} \varepsilon^{0, \mu},
$$

and the wave function $\Psi(x)$ is the same as in Eq.(7). The Hermitianizing matrix, obeying equations: $\eta \Gamma_{i}=-\Gamma_{i}^{+} \eta^{+}, \eta \Gamma_{4}=\Gamma_{4}^{+} \eta^{+}(i=1,2,3)$ is given 
by

$$
\eta=-\frac{m_{0}\left(m+m_{0}\right)}{m^{2}} \varepsilon^{0,0}+\frac{m+m_{0}}{m_{0}}\left(\varepsilon^{m, m}-\varepsilon^{4,4}+\varepsilon^{[m 4],[m 4]}-\frac{1}{2} \varepsilon^{[m n],[m n]}\right) .
$$

The "conjugated" wave function $\bar{\Psi}(x)=\Psi^{+}(x) \eta$ obeys the equation as follows:

$$
\bar{\Psi}(x)\left(\Gamma_{\mu} \overleftarrow{\partial}_{\mu}-M\right)=0
$$

In the momentum space Eq.(10) becomes

$$
(i \hat{p} \pm M) U_{s}( \pm p)=0
$$

where $\hat{p}=\Gamma_{\mu} p_{\mu}$. Index $s$ in Eq.(14) corresponds to the state with definite spin (one and zero) and spin projections (for spin-1). One can verify that the matrix $\hat{p}$ satisfies the minimal equation

$$
\hat{p}^{5}-\frac{\left(m^{2}+m_{0}^{2}\right) p^{2}}{\left(m+m_{0}\right)^{2}} \hat{p}^{3}+\frac{M^{2} p^{4}}{\left(m+m_{0}\right)^{2}} \hat{p}=0 .
$$

The squared of four-momentum $p^{2}$ is not specified here and obeys the dispersion equation (3). Eq.(15) can be cast also in the form

$$
\hat{p}\left(\hat{p}^{2}-\frac{M^{2}}{m_{0}^{2}} p^{2}\right)\left(\hat{p}^{2}-\frac{M^{2}}{m^{2}} p^{2}\right)=0 .
$$

Equations (15), (16) allow us to obtain the density matrix. With the help of equations (15), (16), one can verify that the projection matrix

$$
\rho( \pm p)=\frac{\left(m+m_{0}\right)^{4} i \hat{p}(i \hat{p} \mp M)}{2 m^{2} m_{0}^{2}\left(p^{4}-m^{2} m_{0}^{2}\right)}\left[\hat{p}^{2}+\frac{p^{4}}{\left(m+m_{0}\right)^{2}}\right]
$$

obeys the equations

$$
\begin{gathered}
(i p \pm M) \rho( \pm p)=0, \\
\rho( \pm p)^{2}=\rho( \pm p), \quad \rho(+p) \rho(-p)=0 .
\end{gathered}
$$

The projection matrix (17) is the density matrix for impure spin states:

$$
(\rho( \pm p))_{A B}=\sum_{s}\left(U_{s}( \pm p)\right)_{A}\left(\bar{U}_{s}( \pm p)\right)_{B},
$$


where the $U_{s}( \pm p)$ is the wave function for the pure spin state and obeys Eq.(14). It should be noted that the density matrix (17) obtained obeys Eq.(18) for the arbitrary momentum $p_{\mu}$ satisfying the dispersion equation (3). Contrarily, in [3], we found the density matrices only for the case specifying the four-momentum: for the vector state $p^{2}=-m^{2}$ and for the scalar state $p^{2}=-m_{0}^{2}$. Thus, we can introduce the quantum number $\tau=0,1$, that $p^{2}=-m_{\tau}^{2}$ (no summation in index $\tau$ ). It is implied that four momentum $p=\left(\mathbf{p}, i p_{0}\right)$ possesses the additional quantum number $\tau=0,1$ corresponding to the mass $m_{0}$ and $m \equiv m_{1}$.

From the Lagrangian

$$
\mathcal{L}=-\frac{1}{2}\left[\bar{\Psi}(x)\left(\Gamma_{\mu} \partial_{\mu}+M\right) \Psi(x)-\bar{\Psi}(x)\left(\Gamma_{\mu} \overleftarrow{\partial}_{\mu}-M\right) \Psi(x)\right]
$$

we obtain the momenta

$$
\begin{gathered}
\pi(x)=\frac{\partial \mathcal{L}}{\partial\left(\partial_{0}\right) \Psi(x)}=\frac{i}{2} \bar{\Psi}(x) \Gamma_{4}, \\
\bar{\pi}(x)=\frac{\partial \mathcal{L}}{\partial\left(\partial_{0}\right) \bar{\Psi}(x)}=-\frac{i}{2} \bar{\Psi}(x) \Gamma_{4} .
\end{gathered}
$$

The Hamiltonian (energy) density is defined as follows:

$$
\begin{gathered}
\mathcal{H}=\pi(x) \partial_{0} \Psi(x)+\left(\partial_{0} \bar{\Psi}(x)\right) \bar{\pi}(x)-\mathcal{L} \\
=\frac{i}{2} \bar{\Psi}(x) \Gamma_{4} \partial_{0} \Psi(x)-\frac{i}{2}\left(\partial_{0} \bar{\Psi}(x)\right) \Gamma_{4} \Psi(x) .
\end{gathered}
$$

It is easy to verify that $\mathcal{H}=T_{44}$, where

$$
T_{\mu \nu}^{c}=\frac{1}{2}\left(\partial_{\nu} \bar{\Psi}(x)\right) \Gamma_{\mu} \Psi(x)-\frac{1}{2} \bar{\Psi}(x) \Gamma_{\mu} \partial_{\nu} \Psi(x)
$$

is the canonical energy-momentum tensor. One may verify, with the help of Eq.(10),(13), that this tensor is conserved: $\partial_{\mu} T_{\mu \nu}^{c}=0$.

\section{Quantization}

From the standard relation $\left[\Psi_{M}(\mathbf{x}, t), \pi_{N}(\mathbf{y}, t)\right]=i \delta_{M N} \delta(\mathbf{x}-\mathbf{y})$, we arrive, using Eq.(22), at simultaneous quantum commutators

$$
\left[(\Psi(\mathbf{x}, t))_{M},\left(\bar{\Psi}(\mathbf{y}, t) \Gamma_{4}\right)_{N}\right]=2 \delta_{M N} \delta(\mathbf{x}-\mathbf{y}) .
$$


From Eq.(25),(7),(11), one obtains non-zero field commutators:

$$
\left[B^{*}(\mathbf{x}, t), B_{4}(\mathbf{y}, t)\right]=2 m \delta(\mathbf{x}-\mathbf{y}), \quad\left[B_{[m 4]}(\mathbf{x}, t), B_{n}^{*}(\mathbf{y}, t)\right]=2 m \delta_{m n} \delta(\mathbf{x}-\mathbf{y}) .
$$

Let us consider solutions to Eq.(10) with definite spin, spin projections, energy and momentum for two mass states in the form of plane waves:

$$
\Psi_{s, \tau}^{( \pm)}(x)=\sqrt{\frac{m_{\tau}^{2}}{p_{0} V M}} U_{s, \tau}( \pm p) \exp ( \pm i p x),
$$

where $V$ is the normalization volume. We use the normalization conditions

$$
\int_{V} \bar{\Psi}_{s, \tau}^{( \pm)}(x) \Gamma_{4} \Psi_{s^{\prime}, \tau}^{( \pm)}(x) d^{3} x= \pm \delta_{s s^{\prime}}, \quad \int_{V} \bar{\Psi}_{s, \tau}^{( \pm)}(x) \Gamma_{4} \Psi_{s^{\prime}, \tau}^{(\mp)}(x) d^{3} x=0,
$$

where $\bar{\Psi}_{s, \tau}^{( \pm)}(x)=\left(\Psi_{s, \tau}^{( \pm)}(x)\right)^{+} \eta$. With the help of normalization conditions (28), one obtains relations for the functions $U_{s, \tau}( \pm p)$ :

$$
\begin{gathered}
\bar{U}_{s, \tau}( \pm p) \Gamma_{\mu} U_{s^{\prime}, \tau}( \pm p)=\mp \frac{i M p_{\mu}}{m_{\tau}^{2}} \delta_{s s^{\prime}}, \quad \bar{U}_{s, \tau}( \pm p) U_{s^{\prime}, \tau}( \pm p)=\delta_{s s^{\prime}} \\
\bar{U}_{s, \tau}( \pm p) \Gamma_{4} U_{s^{\prime}, \tau}(\mp p)=0 .
\end{gathered}
$$

In the second quantized theory the field operator may be written as

$$
\Psi_{\tau}(x)=\sum_{p, s}\left[a_{s, p} \Psi_{s, \tau}^{(+)}(x)+b_{s, p}^{+} \Psi_{s, \tau}^{(-)}(x)\right],
$$

where positive and negative parts of the wave function are defined by Eq.(27). The creation and annihilation operators of particles $a_{s, p}^{+}, a_{s, p}$ and antiparticles $b_{s, p}^{+}, b_{s, p}$ satisfy the commutation relations:

$$
\begin{gathered}
{\left[a_{s, p}, a_{s^{\prime}, p^{\prime}}^{+}\right]=\delta_{s s^{\prime}} \delta_{p p^{\prime}}, \quad\left[b_{s, p}, b_{s^{\prime}, p^{\prime}}^{+}\right]=\delta_{s s^{\prime}} \delta_{p p^{\prime}},} \\
{\left[a_{s, p}, a_{s^{\prime}, p^{\prime}}\right]=\left[b_{s, p}, b_{s^{\prime}, p^{\prime}}\right]=\left[a_{s, p}, b_{s^{\prime}, p^{\prime}}\right]=\left[a_{s, p}, b_{s^{\prime}, p^{\prime}}^{+}\right]=0 .}
\end{gathered}
$$

With the help of Eq.(27),(28),(30),(31), one obtains the Hamiltonian

$$
H=\int_{V} \mathcal{H} d^{3} x=\sum_{s, p} p_{0}\left(a_{s, p}^{+} a_{s, p}+b_{s, p} b_{s, p}^{+}\right)
$$

We find from Eq.(30),(31) commutation relations as follows:

$$
\left[\Psi_{\tau M(x)}, \Psi_{\tau N}\left(x^{\prime}\right)\right]=\left[\bar{\Psi}_{\tau M}(x), \bar{\Psi}_{\tau N}\left(x^{\prime}\right)\right]=0,
$$




$$
\begin{gathered}
{\left[\Psi_{\tau M}(x), \bar{\Psi}_{\tau N}\left(x^{\prime}\right)\right]=N_{\tau M N}\left(x, x^{\prime}\right),} \\
N_{\tau M N}\left(x, x^{\prime}\right)=N_{\tau M N}^{+}\left(x, x^{\prime}\right)-N_{\tau M N}^{-}\left(x, x^{\prime}\right), \\
N_{\tau M N}^{+}\left(x, x^{\prime}\right)=\sum_{s, p}\left(\Psi_{s, \tau}^{(+)}(x)\right)_{M}\left(\overline{\Psi_{s, \tau}^{(+)}}\left(x^{\prime}\right)\right)_{N}, \\
N_{\tau M N}^{-}\left(x, x^{\prime}\right)=\sum_{s, p}\left(\Psi_{s, \tau}^{(-)}(x)\right)_{M}\left(\overline{\Psi_{s, \tau}^{(-)}}\left(x^{\prime}\right)\right)_{N} .
\end{gathered}
$$

From Eq.(30), one arrives at

$$
N_{\tau M N}^{ \pm}\left(x, x^{\prime}\right)=\sum_{s, p} \frac{m_{\tau}^{2}}{p_{0} V M}\left(U_{s, \tau}( \pm p)\right)_{M}\left(\bar{U}_{s, \tau}( \pm p)\right)_{N} \exp \left[ \pm i p\left(x-x^{\prime}\right)\right],
$$

Taking into account Eq.(17),(20), and the relation $p^{2}=-m_{\tau}^{2}$, from Eq.(35), we obtain:

$$
\begin{gathered}
N_{\tau M N}^{ \pm}\left(x, x^{\prime}\right)=\sum_{p}\left\{\frac{i\left(m+m_{0}\right)^{4} m_{\tau}^{2} \widehat{p}(i \widehat{p} \mp M)}{2 p_{0} V M m^{2} m_{0}^{2}\left(m_{\tau}^{4}-m^{2} m_{0}^{2}\right)}\left[\widehat{p}^{2}+\frac{m_{\tau}^{4}}{\left(m+m_{0}\right)^{2}}\right]\right\}_{M N} \\
\times \exp \left[ \pm i p\left(x-x^{\prime}\right)\right]=\left\{\frac{\left(m+m_{0}\right)^{4} m_{\tau}^{2}\left( \pm \Gamma_{\mu} \partial_{\mu}\right)\left( \pm \Gamma_{\mu} \partial_{\mu} \mp M\right)}{M m^{2} m_{0}^{2}\left(m_{\tau}^{4}-m^{2} m_{0}^{2}\right)}\right. \\
\left.\times\left[\frac{m_{\tau}^{4}}{\left(m+m_{0}\right)^{2}}-\left(\Gamma_{\mu} \partial_{\mu}\right)^{2}\right]\right\}_{M N} \sum_{p} \frac{1}{2 p_{0} V} \exp \left[ \pm i p\left(x-x^{\prime}\right)\right] .
\end{gathered}
$$

Using the singular functions [6]

$$
\begin{gathered}
\Delta_{+}(x)=\sum_{p} \frac{1}{2 p_{0} V} \exp (i p x), \quad \Delta_{-}(x)=\sum_{p} \frac{1}{2 p_{0} V} \exp (-i p x), \\
\Delta_{0}(x)=i\left(\Delta_{+}(x)-\Delta_{-}(x)\right),
\end{gathered}
$$

one finds from Eq.(35),(37) the equation

$$
\begin{gathered}
N_{\tau M N}\left(x, x^{\prime}\right)=-i\left\{\frac{\left(m+m_{0}\right)^{4} m_{\tau}^{2}\left(\Gamma_{\mu} \partial_{\mu}\right)\left(\Gamma_{\mu} \partial_{\mu}-M\right)}{M m^{2} m_{0}^{2}\left(m_{\tau}^{4}-m^{2} m_{0}^{2}\right)}\right. \\
\left.\times\left[\frac{m_{\tau}^{4}}{\left(m+m_{0}\right)^{2}}-\left(\Gamma_{\mu} \partial_{\mu}\right)^{2}\right]\right\}_{M N} \Delta_{0}\left(x-x^{\prime}\right) .
\end{gathered}
$$


If the points $x$ and $x^{\prime}$ are separated by the space-like interval $\left(x-x^{\prime}\right)>0$, the commutator $\left[\Psi_{M}(x), \bar{\Psi}_{N}\left(x^{\prime}\right)\right]$ vanishes due to the properties of the function $\Delta_{0}(x)$ [6]. With the help of Eq.(15), one may verify that the equation

$$
\left(\Gamma_{\mu} \partial_{\mu}+M\right) N_{\tau}^{ \pm}\left(x, x^{\prime}\right)=0 .
$$

is valid. The vacuum expectation of chronological pairing of operators (the propagator) is defined as follows:

$$
\begin{gathered}
\left\langle T \Psi_{\tau M}(x) \bar{\Psi}_{\tau N}(y)\right\rangle_{0}=N_{\tau M N}^{c}(x-y) \\
=\theta\left(x_{0}-y_{0}\right) N_{\tau M N}^{+}(x-y)+\theta\left(y_{0}-x_{0}\right) N_{\tau M N}^{-}(x-y),
\end{gathered}
$$

where $\theta(x)$ is the theta-function. From Eq.(37), we obtain

$$
\begin{gathered}
\left\langle T \Psi_{\tau M}(x) \bar{\Psi}_{\tau N}(y)\right\rangle_{0}=\left\{\frac{\left(m+m_{0}\right)^{4} m_{\tau}^{2}\left(\Gamma_{\mu} \partial_{\mu}\right)\left(\Gamma_{\mu} \partial_{\mu}-M\right)}{M m^{2} m_{0}^{2}\left(m_{\tau}^{4}-m^{2} m_{0}^{2}\right)}\right. \\
\left.\times\left[\frac{m_{\tau}^{4}}{\left(m+m_{0}\right)^{2}}-\left(\Gamma_{\mu} \partial_{\mu}\right)^{2}\right]\right\}_{M N} \Delta_{c}(x-y) .
\end{gathered}
$$

The function $\Delta_{c}(x-y)$ is given by

$$
\Delta_{c}(x-y)=\theta\left(x_{0}-y_{0}\right) \Delta_{+}(x-y)+\theta\left(y_{0}-x_{0}\right) \Delta_{-}(x-y) .
$$

It follows from Eq.(41) that the propagator with $\tau=0$ possesses the opposite sign compared with the case $\tau=1$. This confirms that the state of the field with spin-0 is the ghost.

\section{Conclusion}

We have considered the fields possessing two masses, $m, m_{0}$ with spin one and zero, i.e multi-spin 1,0. The propagators of fields in the second order and first order formulations are obtained. We found the density matrix and performed canonical quantization of bosonic fields in the first order formalism. The commutation relations and the vacuum expectation of chronological pairing of operators obtained allow us to investigate quantum processes with bosonic fields. It should be stressed also that ghosts (k-essence, Phantom ) are widely used in modern cosmology [7]. Therefore, the presence of spin- 0 state of the fields may be used in some cosmological models. 


\section{References}

[1] I. L. Buchbinder, S. D. Odintsov, I. L. Shapiro, Effective action in quantum gravity (IOP Publishing Ltd., Btistol, UK, 1992).

[2] S. I. Kruglov, Annales Fond. Broglie 32 (2007), no 2-3 [arXiv: 0710.2534 (gr-qc)].

[3] S. I. Kruglov, Int. J. Mod. Phys. A16, 4925 (2001) [arXiv: hep-th/0110083.

[4] S. I. Kruglov, Symmetry and Electromagnetic Interaction of Fields with Multi-Spin (Nova Science Publishers, Huntington, New York, 2001).

[5] H. Ruegg and M. Ruiz-Altaba, Int. J. Mod. Phys. A19, 3265 (2004) [arXiv: hep-th/0304245].

[6] A. I. Ahieser and V. B. Berestetskii, Quantum Electrodynamics (New York: Wiley Interscience, 1969).

[7] V. Mukhanov, Physical Foundations of Cosmology (Cambridge University Press, 2005, 421 p.). 